\title{
Editorials
}

\section{Ultrasound-guidance and nerve stimulation: implications for the future practice of regional anesthesia}

\author{
Ban Tsui MSc MD FRCPC
}

A $\mathrm{N}$ ever increasing demand for regional anesthesia from both patients and surgeons matches the growing realization that both peripheral and neuraxial blockade can provide superior pain management and perhaps improve patient outcomes, to meet evolving expectations for ambulatory, cost-effective surgery. ${ }^{1,2}$ Our aging population presents with an increasing range of co-morbidities, demanding a wider choice of surgical anesthesia options, including the use of a variety of regional techniques in conjunction with general anesthesia, to optimize clinical care, while at the same time reducing the risks of complications.

Regional anesthesia with single-shot or continuous peripheral nerve blocks (PNB) can provide superior analgesia and a lower incidence of side effects compared to parenteral anesthetics (e.g., patient-controlled opioid analgesia). ${ }^{3-5}$ However, both single shot and continuous techniques are highly dependent on the accurate delivery of a correct dose of local anesthetic to attain success, and to avoid rare but potentially devastating nerve damage. ${ }^{6,7}$ This dependency remains a concern, particularly as many anesthesia training programs are not providing the necessary resources for practitioners to develop the requisite skills and confidence with regional anatomy to performing $\mathrm{PNBs}$ on a routine basis., ${ }^{8}$ One of the principal challenges of regional anesthesia is the unreliability of conventional modalities for confirming precise nerve localization. Such conventional modalities include electrical stimulation (ES) and patientreported paresthesiae which rely on surface landmark identification. Both techniques are highly dependent on inter-individual anatomical and physiological varia- tions and, in the case of ES, equipment accuracy. ${ }^{10-12}$ Thus, despite its scientific foundations, the practice of regional anesthesia remains an "art" for many practitioners, and consistent success with these techniques often appears to be limited to anesthesiologiosts who are regional anesthesia enthusiasts.

Nevertheless, the introduction some 30 years ago of an objective means for identifying needle-nerve proximity, ES, was an integral step towards transforming regional anesthesia into a "science". Nerve stimulation, however, also has its limitations. The technique relies on physiological responses of neural structures to $\mathrm{ES}$, for which there is considerable inter-individual variation. Accordingly, it is fitting to question the significance of the commonly accepted threshold current of $0.5 \mathrm{~mA}$ to ensure block success, when a range of current thresholds more realistically reflects normal physiological variability. Furthermore, ES responses are influenced by the interplay of injectates, physiologic solutions (e.g., blood) and disease. Not surprisingly, the introduction of ES failed to result in a renewed interest in regional anesthesia.

One of the most exciting recent technological advances in this field has been the introduction of anatomically-based ultrasound (US) imaging. The introduction of this technology represents the first time in nearly 100 years of the practice of regional anesthesia, that an operator has been able to view an image of the target nerve. While ultrasound guidance is sure to draw many anesthesiologists back towards the practice of regional anesthesia, its more widespread use will ultimately depend on its proven clinical efficacy, cost-effectiveness and practicality. In this context, the study reported in this issue of the Journal by Chan et

CAN J ANESTH $2007 / 54: 3 /$ pp 165-170

From the Department of Anesthesiology and Pain Medicine, University of Alberta Hospitals, Edmonton, Alberta, Canada.

Address correspondence to: Dr. Ban Tsui, Department of Anesthesiology and Pain Medicine, 8-120 Clinical Sciences Building, University of Alberta Hospitals, Edmonton, Alberta T6G 2G3, Canada. Phone: 780-407-8861; Fax: 780-407-3200; E-mail: btsui@ualberta.ca 
al. provides new insights of relevance to all anesthesiologists. ${ }^{13}$ In a large, randomized controlled trial, the authors compared the success rate of US-guided axillary blockade compared to an ES technique alone or in combination with US, in patients undergoing upper limb surgery. Block success rates were found to be significantly higher in the US and US+ES groups compared to ES alone, while conferring additional benefits of less postoperative axillary bruising and pain. ${ }^{13}$ While these findings might suggest that US alone may be able to replace an ES technique, there are several issues which must be considered to appreciate the complexities of introducing this technology into routine clinical practice.

Studies comparing US imaging and ES techniques for upper extremity plexus anesthesia have demonstrated the superiority of US with respect to: block completeness at $30 \mathrm{~min},{ }^{13,14}$ overall block success (surgical anesthesia) ${ }^{13,15}$ reduced block performance and onset times, ${ }^{13-17}$ prolongation of block ${ }^{16}$ and reduced complications. ${ }^{18}$ While the cumulative evidence may appear very convincing, many of the studies show conflicting results for certain parameters, and the large variability in trial methodologies and application of different outcome measures account for many of the discrepancies. Indeed, the various endpoints used during research in regional anesthesia may bias outcomes when comparing multiple regional techniques. This matter is evident in two of the results generated by Chan et al. ${ }^{13}$ First, the US-guided block success rate was lower than anticipated $(80-83 \%)$ due to rigid definitive endpoints of complete pinprick anesthesia in three nerves at $30 \mathrm{~min}$. Secondly, their failure to show a benefit of combining US and ES may have resulted from using visualized circumferential injectate spread to determine injection, rather than an acceptable motor response within a certain milliamperage range. Conversely, their endpoints for using ES, which accepted a triceps muscle contraction for radial nerve involvement, may have been detrimental to the block onset timing with this technique, as well as success rates, as these proximal responses may have been inadequate to produce optimal results. This is especially true considering that the radial nerve is often the most difficult nerve to locate during axillary blockade; clearly, US visibility of the nerve will enhance success.

However, one must be mindful of the fact that, despite the initial excitement of this technique, US visualization is still indirect, and images are subject to individual interpretation. In fact, it is not surprising that Chan's group were unable to demonstrate an added benefit when combining ES and US, as this group of investigators is very experienced with both regional techniques. Rather than abandoning ES techniques in favour of US imaging alone, a more prudent course might be to combine the two technologies to further approach the goal of a $100 \%$ success rate, without untoward side effects, for all regional blocks. Ultrasound guidance, like ES, is not perfect. We can never be totally sure of a nerve's identity, as even Chan's group, using high-end ultrasound technology failed to achieve $100 \%$ block success in the ultrasound guided groups. They commented that: "this is likely the result of mistaken nerve identity in Group US and misinterpretation of local anesthetic circumferential spread..."13

Apart from block success, cost-effectiveness and practicality need to be demonstrated in order to fully support US guidance in regional anesthesia. Ultrasound may not reduce costs if nerve stimulation is still required to provide additive confirmation of needle-nerve proximity, or for the initial learning stages of US-guidance. On the other hand, routine application of this technology may ultimately increase the overall utilization rates of regional anesthesia. Maximizing block accuracy ${ }^{6,19,20}$ as well as improving training for those inexperienced in regional techniques $^{2,21}$ are both attractive incentives for using US. Importantly, US guidance may improve two critical factors related to trainees' comfort with peripheral nerve blocks: familiarity with the relevant anatomy, and confidence level with the block, through visualization of related anatomical structures, needle trajectory and local anesthetic application. ${ }^{2,21}$

Regardless of either real or perceived advantages, there are some limitations to note. Some anesthesiologists are expert in their ability to interpret US images, while many others have more limited experience. Ultrasonography for regional anesthesia can have a long learning curve, especially when considering the variables of controlling and tracking needle insertion. Indeed, in their study of simulated US-guidance with residents, Sites et al..$^{22}$ identified a "concerning novice pattern" where residents failed to adequately view their block needle at all times during the procedure. Continual identification of the block needle is essential for improvements in success and safety of peripheral blocks, particularly in sensitive sites such as the infraclavicular block location ${ }^{23}$ or when traditional landmarks are not used for needle insertion (i.e., US guidance is used in a novel manner). Developing methods to best align the probe and needle, and to optimize needle visibility within the tissues, will help with training and general performance of US guidance. Additionally, developing systematic scanning 
methods to reliably identify the target nerves will be particularly beneficial to novice operators. Including ES for objective determination of the nerve's identity will be useful, particularly for clinicians or residents first learning the technique of US guidance. Finally, it will be important to develop unique training standards to guide residency training programs and teaching institutions, and to establish evaluation criteria for performing US-guided regional anesthesia.

A final issue which is particularly relevant to US guided regional anesthesia relates to the very practical aspects of performing blocks in this manner. Many fine studies showing success of the technique ${ }^{13}$ or reviewing the methodology ${ }^{20}$ of US guided blocks used very high quality cart-based US systems (e.g., Philips HDI 5000, Philips Medical Systems ATL Ultrasound, Bothell, WA, USA), which provide compound imaging, and generate high resolution images where anatomical and block substances can be visualized very clearly. My experience with the more affordable and more compact machines (Titan, SonoSite Inc., Bothell, WA, USA) demonstrates a need for considerable learning to successfully use, and train others with these systems.

Further evidence-based research will certainly improve this specialty's view of US-guided nerve blocks, and ultimately, the practice of regional anesthesia. In this regard, Chan et al.'s excellent study will influence our approach to, and consideration of USguided regional anesthesia. ${ }^{13}$ While the direct costs of US vs ES, or block performance times may be easy to compare, the true cost-benefits for routine application of this technology in clinical practice will require further evaluation, particularly for ambulatory surgery. The emerging literature on teaching and training in US-guided nerve blocks will establish evidencebased approaches to determine the most effective applications for this "state of the art" technology. In addition to reports from well-conducted randomized controlled trials, subtle but critical changes are likely to be seen through observation of resident training in PNBs, and increasing our specialty's clinical experience with US for regional anesthesia. Ultimately, patient satisfaction with regional anesthesia is likely to increase, and the benefits of US-guided nerve blocks will become evident to the full spectrum of health care professionals.

\section{Échoguidage et neuro- stimulation : implica- tions pour la pratique future de l'anesthésie régionale}

L'augmentation de la demande des techniques d'anesthésie régionale, de la part des patients aussi bien que des chirurgiens, est issue de la prise de conscience progressive que les blocs périphériques et neuraxiaux permettent un meilleur soulagement de la douleur; de plus, ils semblent améliorer l'évolution des patients, permettant ainsi d'atteindre les objectifs coût-bénéfices contemporains de la chirurgie ambulatoire. ${ }^{1,2}$ Une population vieillissante avec un nombre et une variété croissants de co-morbidités nécessite un choix plus grand d'options d'anesthésie chirurgicale, y compris l'utilisation d'une diversité de techniques régionales en conjonction avec l'anesthésie générale et ce, afin d'optimiser les soins cliniques tout en réduisant les risques de complications.

L'anesthésie régionale effectuée par blocs nerveux périphériques (BNP) à injection unique ou avec perfusion continue peuvent fournir une meilleure analgésie avec moins d'effets secondaires que les anesthésiques parentéraux (par ex., l'analgésie avec opiacés contrôlée par le patient). ${ }^{3-5}$ Toutefois, la réussite des techniques à injection unique et en perfusion dépend fortement de l'administration précise d'une dose adéquate d'anesthésique local, tout en évitant les lésions neurales rares mais potentiellement dangereuses. ${ }^{6,7}$ Cette dépendance demeure une préoccupation, particulièrement du fait que nombre de programmes de formation en anesthésie ne fournissent pas les ressources nécessaires aux médecins pour qu'ils puissent développer les compétences requises ainsi qu'une aisance avec l'anatomie régionale qui leur permettrait d'effectuer des BNP de façon routinière. ${ }^{8,9}$ Un des principaux défis de l'anesthésie régionale est le manque de fiabilité des modalités conventionnelles dans la localisation précise des nerfs. Ces modalités conventionnelles sont la stimulation électrique (SE) et la paresthésie rapportée par le patient, qui se basent sur l'identification de signes de surface. Les deux techniques dépendent fortement des variations anatomiques et physiologiques entre individus, et, dans le cas de la SE, de la précision de l'équipement. ${ }^{10-12}$ Ainsi, malgré ses fondements sci- 
entifiques, la pratique de l'anesthésie régionale reste un «art » pour de nombreux médecins, et une constance dans la réussite de ces techniques est souvent décrite comme l'apanage d'anesthésistes passionnés d'anesthésie régionale.

Il y a une trentaine d'années, l'introduction d'un moyen objectif d'évaluer la proximité entre l'aiguille et le nerf, la SE, a toutefois été un pas important dans la transformation progressive de l'anesthésie régionale en «science». Cependant, la neurostimulation a elle aussi ses limites. La technique s'appuie sur les réactions physiologiques des structures nerveuses à la SE, qui varient grandement d'un individu à l'autre. Dès lors, on peut remettre en question la signification du seuil communément accepté de courant électrique à $0,5 \mathrm{~mA}$ pour assurer la réussite du bloc; une gamme de seuils de courant reflèterait de façon plus réaliste les variations physiologiques normales. De plus, les réactions à la SE sont influencées par l'interaction entre les produits injectés, les solutions physiologiques (par ex. du sang) et la maladie. Comme on pouvait s'y attendre, l'introduction de la SE n'a pas provoqué de renouveau d'intérêt pour l'anesthésie régionale.

L'introduction de l'imagerie échographique basée sur l'anatomie a été l'un des progrès technologiques récents les plus intéressants dans ce domaine. Grâce à l'introduction de cette technologie, c'est la première fois en presque cent ans de pratique d'anesthésie régionale qu'un opérateur peut voir une image du nerf ciblé. Bien que l'échographie de guidage fasse certainement revenir de nombreux anesthésiologistes vers la pratique de l'anesthésie régionale, son utilisation plus répandue dépendra en fin de compte de son efficacité, son rapport coût-bénéfice et de son aspect pratique. Dans ce contexte, l'étude de Chan et coll. dans ce numéro du Journal offre de nouvelles perspectives pour tous les anesthésiologistes. ${ }^{13}$ Dans leur vaste étude, randomisée et contrôlée, les auteurs ont comparé le taux de succès de blocs axillaires guidés par échographie à une technique de SE seule ou combinée à un échoguidage (EG) chez des patients subissant une chirurgie d'un membre supérieur. Ils ont observé un taux de succès des blocs significativement plus élevé dans les groupes EG et EG + ES que dans le groupe ES seule, et également des bienfaits supplémentaires, notamment moins d'hématomes et de douleurs axillaires après l'opération. ${ }^{13}$ Bien que ces constatations suggèrent que l'utilisation de l'échographie seule puisse remplacer une technique de SE, plusieurs considérations doivent être prises en compte afin d'apprécier la complexité d'une introduction de cette technique dans la pratique clinique de tous les jours.
Les études comparant l'imagerie EG et les techniques de SE pour l'anesthésie du plexus d'un membre supérieur ont démontré que l'échographie est supérieure quant à : l'étendue du bloc après $30 \mathrm{~min},{ }^{13,14}$ la réussite globale du bloc (anesthésie chirurgicale), ${ }^{13,15}$ un temps réduit de réalisation du bloc et de son installation, ${ }^{13,17}$ la durée du bloc ${ }^{16}$ et la réduction des complications. ${ }^{18}$ Quoique les preuves accumulées puissent paraître très convaincantes, nombre de ces études indiquent des résultats contradictoires pour certains paramètres; de plus, l'importante variabilité des méthodologies d'études ainsi que l'application de différentes mesures de résultat expliquent la plupart des divergences. En effet, les divers critères d'évaluation utilisés pendant la recherche en anesthésie régionale peuvent biaiser les résultats lors de la comparaison de plusieurs techniques régionales. Ceci est évident pour deux des résultats observés par Chan et coll. ${ }^{13}$ En premier lieu, le taux de réussite des blocs guidés par EG était plus bas que prévu (80-83\%) à cause de critères rigides d'évaluation de l'anesthésie complète à la piqûre d'aiguille dans trois territoires nerveux à $30 \mathrm{~min}$. Deuxièmement, le fait qu'ils n'aient pas pu démontrer l'avantage de combiner l'EG et la SE pourrait provenir de l'utilisation d'une couche circonférentielle visualisée de produit injecté afin de déterminer l'injection plutôt qu'une réponse motrice acceptable dans une fourchette de milli ampérage déterminée. Inversement, leurs critères concernant l'utilisation de SE, qui englobait une contraction du triceps dans une implication du nerf radial, ont peutêtre nui à la mesure du temps de début du bloc avec cette technique aussi bien qu'aux taux de réussite, étant donné que ces réactions proximales ont pu être inadéquates à produire des résultats optimaux. Ceci est particulièrement vrai si l'on prend en compte le fait que le nerf radial est souvent le plus difficile à localiser pendant un bloc axillaire; il est clair que la visibilité échographique du nerf améliorera la réussite.

Toutefois, il ne faut pas oublier que, malgré l'emballement initial suscité par cette technique, la visualisation échographique demeure indirecte, et les images sont sujettes à l'interprétation individuelle. En fait, il n'est pas surprenant que le groupe de Chan n'ait pas réussi à démontrer un avantage supplémentaire lors de la combinaison de SE et d'EG, étant donné que le groupe de chercheurs en question a une grande expérience des deux techniques régionales. Plutôt que d'abandonner les techniques de SE au profit de l'EG seule, une approche plus prudente combinerait les deux technologies afin de progresser vers l'objectif d'un taux de réussite de $100 \%$ pour tous les blocs régionaux, sans effets secondaires fầcheux. L'échoguidage, tout comme 
la SE, n'est pas parfait. Nous ne pouvons jamais être complètement certains de l'identité d'un nerf, comme l'a démontré le groupe de Chan, qui n'a pas atteint un taux de succès de bloc à $100 \%$ dans les groupes d'échographie de guidage et ce, même en utilisant une technologie échographique haut de gamme. Ils remarquent : "Ceci est probablement la conséquence d'une identification erronée du nerf dans le groupe EG et d'une mauvaise interprétation de l'étendue circonférentielle de l'anesthésique local... »13

Outre la réussite du bloc, le rapport coût-bénéfice et l'aspect pratique doivent être démontrés afin d'appuyer complètement l'utilisation de l'échoguidage pour l'anesthésie régionale. L'échographie pourrait ne pas réduire les coûts si la stimulation nerveuse est encore requise afin de confirmer la proximité aiguillenerf, ou lors des étapes initiales d'apprentissage de l'échoguidage. Néanmoins, l'application régulière de cette technologie pourrait en définitive augmenter les taux globaux d'utilisation de l'anesthésie régionale. Deux incitatifs importants à l'utilisation de l'EG sont la maximisation de la précision du bloc $^{6,19,20}$ et l'amélioration de la formation pour ceux qui ne connaissent pas les techniques régionales.,21 Particulièrement, l'échoguidage pourrait améliorer deux facteurs critiques liés au confort des stagiaires par rapport aux blocs nerveux : une certaine familiarité avec l'anatomie en question, et un niveau d'aisance par rapport au bloc grâce à la visualisation des structures anatomiques adjacentes, de la trajectoire de l'aiguille et de l'application de l'anesthésique local.,21

Indépendamment des avantages réels ou perçus, certaines limites sont à noter. Certains anesthésiologistes sont experts dans leur capacité à interpréter les images EG, alors que beaucoup ont une expérience plus limitée. L'échographie pour l'anesthésie régionale peut présenter une longue courbe d'apprentissage, surtout si l'on tient compte des variables quant au contrôle et au suivi de l'insertion de l'aiguille. En effet, dans leur étude sur l'échoguidage simulé chez des résidents, Sites et coll. ${ }^{22}$ ont identifié une "inquiétante tendance de novice ", décrivant ainsi le fait que les résidents ne parvenaient pas à voir correctement leur aiguille et ce, en tout temps durant la procédure. L'identification continue de l'aiguille est essentielle pour améliorer la réussite et la sécurité des blocs nerveux, particulièrement dans des sites sensibles comme la localisation d'un bloc infraclaviculaire ${ }^{23}$ ou lorsque les repères traditionnels ne sont pas utilisés pour l'insertion de l'aiguille (i.e., l'échoguidage est utilisé d'une nouvelle manière).

Le développement de méthodes qui permettent d'aligner au mieux la sonde et l'aiguille ainsi que l'optimisation de la visibilité de l'aiguille dans les tissus aideront à la formation et à la performance générale de l'échoguidage. De plus, le développement de méthodes de balayage systématique afin d'identifier de manière fiable les nerfs ciblés sera particulièrement bénéfique aux novices. L'utilisation de SE pour une détermination objective de l'identité du nerf sera utile, surtout pour les médecins ou résidents apprenant pour la première fois la technique de l'échoguidage. En définitive, il est essentiel de développer des normes de formation spécifiques afin de guider les programmes de formation des résidents ainsi que les institutions d'enseignement, et d'établir des critères d'évaluation de la performance de l'anesthésie régionale par échoguidage.

Un dernier point particulièrement pertinent à l'anesthésie régionale par échoguidage concerne les aspects pratiques de la réalisation de blocs de cette façon. Plusieurs études excellentes qui décrivent la réussite de cette technique ${ }^{13}$ ou rendent compte de la méthodologie ${ }^{20}$ des blocs par échographie de guidage se sont servies de systèmes d'échographie portatifs de très haute qualité (par ex., Philips HDI 5000, Philips Medical Systems ATL Ultrasound, Bothell, WA, USA), qui offrent une imagerie combinée et génèrent des images de haute résolution où les structures anatomiques et les solutions injectées peuvent être clairement visualisées. Mon expérience avec des machines meilleur marché et plus compactes (Titan, SonoSite Inc., Bothell, WA, USA) démontre qu'un apprentissage important est nécessaire afin d'utiliser au mieux ces systèmes et d'en enseigner l'utilisation aux autres.

Des recherches basées sur des données probantes plus approfondies amélioreront certainement la perception des blocs nerveux par échoguidage par les spécialistes et, en fin de compte, la pratique de l'anesthésie régionale. A cet égard, l'excellente étude de Chan et coll. influencera notre approche et notre perception de l'anesthésie régionale par échoguidage. ${ }^{13}$ Bien que les coûts directs de l'EG vs la SE, ainsi que les temps de réalisation des blocs soient faciles à comparer, le véritable rapport coût-bénéfice de l'application routinière de cette technologie dans la pratique clinique nécessite une évaluation plus approfondie, particulièrement en ce qui concerne la chirurgie ambulatoire. La littérature récente au sujet de l'enseignement et de la pratique des blocs nerveux par échoguidage établira des approches basées sur des données probantes qui permettront de définir les applications les plus efficaces pour cette technologie « dernier cri ». En plus des rapports d'études randomisées et contrôlées bien menées, des changements 
subtils mais non moins cruciaux vont probablement être le résultat de l'observation de l'apprentissage des résidents de la technique des blocs nerveux, ainsi que de notre expérience clinique accrue en l'échographie pour l'anesthésie régionale. En fin de compte, la satisfaction du patient par rapport à l'anesthésie régionale devrait augmenter, et les avantages des blocs nerveux par échoguidage deviendront alors évidents à tous les professionnels de la santé.

\section{References}

1 Nielsen KC, Steele SM. Outcome after regional anaesthesia in the ambulatory setting--is it really worth it? Best Pract Res Clin Anaesthesiol 2002; 16: 145-57.

2 Broking $K$, Waurick $R$. How to teach regional anesthesia. Curr Opin Anaesthesiol 2006; 19: 526-30.

3 Borgeat A, Schappi B, Biasca N, Gerber C. Patientcontrolled analgesia after major shoulder surgery: patient-controlled interscalene analgesia versus patientcontrolled analgesia. Anesthesiology 1997; 87: 1343-7.

4 Greengrass $R A$. Regional anesthesia for ambulatory surgery. Anesthesiol Clin North America 2000; 18: 341-53, vii.

5 Singelyn FJ, Gouverneur JM. Postoperative analgesia after total hip arthroplasty: i.v. PCA with morphine, patient-controlled epidural analgesia, or continuous "3-in-1" block?: a prospective evaluation by our acute pain service in more than 1,300 patients. J Clin Anesth 1999; 11: 550-4.

6 Marhofer P, Greher M, Kapral S. Ultrasound guidance in regional anaesthesia. Br J Anaesth 2005; 94: 7-17.

7 Stark RH. Neurologic injury from axillary block anesthesia. J Hand Surg [Am] 1996; 21: 391-6.

8 Chelly JE, Greger J, Gebhard R, Hagberg CA, AlSamsam T, Khan A. Training of residents in peripheral nerve blocks during anesthesiology residency. J Clin Anesth 2002; 14: 584-8.

9 Kopacz DJ, Neal JM. Regional anesthesia and pain medicine: residency training--the year 2000. Reg Anesth Pain Med 2002; 27: 9-14.

10 De Andres J, Sala-Blanch X. Peripheral nerve stimulation in the practice of brachial plexus anesthesia: a review. Reg Anesth Pain Med 2001; 26: 478-83.

11 Perlas A, Niazi A, McCartney C, Chan V, Xu D, Abbas $S$. The sensitivity of motor response to nerve stimulation and paresthesia for nerve localization as evaluated by ultrasound. Reg Anesth Pain Med 2006; 31: 44550 .

12 Urmey WF, Stanton J. Inability to consistently elicit a motor response following sensory paresthesia during interscalene block administration. Anesthesiology 2002; 96: 552-4.

13 Chan VW, Perlas A, McCartney CJ, Brull R, Xu D,
Abbas $S$. Ultrasound guidance improves success rate of axillary brachial plexus block. Can J Anesth 2007; 54: 176-82.

14 Williams SR, Chouinard P, Arcand G, et al. Ultrasound guidance speeds execution and improves the quality of supraclavicular block. Anesth Analg 2003; 97: 151823.

15 Sites BD, Beach $M L$, Spence BC, et al. Ultrasound guidance improves the success rate of a perivascular axillary plexus block. Acta Anaesthesiol Scand 2006; 50: 678-84.

16 Marhofer P, Sitzwohl C, Greher M, Kapral S. Ultrasound guidance for infraclavicular brachial plexus anesthesia in children. Anaesthesia 2004; 59: 642-6.

17 Soeding PE, Sha S, Royse CE, Marks P, Hoy G, Royse $A G$. A randomized trial of ultrasound-guided brachial plexus anaesthesia in upper limb surgery. Anaesth Intensive Care 2005; 33: 719-25.

18 Lin FC, Lion JT, Tsai YF, et al. Efficacy of ultrasoundguided axillary brachial plexus block: a comparative study with nerve stimulator-guided method. Chang Gung Med J 2005; 28: 396-402.

19 Gray AT. Ultrasound-guided regional anesthesia: current state of the art. Anesthesiology 2006; 104: 368-73, discussion 5A.

20 Sites BD, Brull R. Ultrasound guidance in peripheral regional anesthesia: philosophy, evidence-based medicine, and techniques. Curr Opin Anaesthesiol 2006; 19: 630-9.

21 Grau T, Bartusseck E, Conradi R, Martin E, Motsch J. Ultrasound imaging improves learning curves in obstetric epidural anesthesia: a preliminary study. Can J Anesth 2003; 50: 1047-50.

22 Sites BD, Gallagher JD, Cravero J, Lundberg J, Blike $G$. The learning curve associated with a simulated ultrasound-guided interventional task by inexperienced anesthesia residents. Reg Anesth Pain Med 2004; 29: 544-8.

23 Sandhu NS, Capan LM. Ultrasound-guided infraclavicular brachial plexus block. Br J Anaesth 2002; 89: 254-9. 